\title{
Vitamin D Deficiency and Critical Illness: Correspondence
}

\author{
D. Vijayasekaran ${ }^{1} \cdot$ Shanthi Ramesh $^{1}$
}

Received: 29 January 2016 / Accepted: 2 March 2016 / Published online: 4 April 2016

(C) Dr. K C Chaudhuri Foundation 2016

To the Editor: The pleiotrophic actions of vitamin D and its role in the functioning of the vital organs have been extensively studied in children and adults after the discovery of vitamin D receptors on various cells [1]. The paper published on "Vitamin D deficiency and critical illness" has highlighted some important aspects that need further research [2].

The analysis of several baseline characteristics concluded that children with higher PRISM III scores were found to have greater vitamin D deficiency [3]. If the underlying cause of such critical illness had been analyzed, the study would have thrown more light on the subject and guided further research.

The study found that majority of the children $(83.8 \%)$ admitted in the PICU were vitamin D deficient. Hence whether the assessment of vitamin D levels could be routinely added in the initial investigatory work up of a critically ill child at least, in higher centers needs to be considered.

It has been observed that sick malnourished children had higher vitamin D levels than well nourished children [4]. Whether similar observations were seen in other studies requires clarification.

Shanthi Ramesh

drshanthiramesh@gmail.com

1 Department of Pediatrics, Sree Balaji Medical College and Hospital, Chennai 600 044, Tamil Nadu, India
Vitamin D receptors are increasingly identified in many vital organs and on immunoregulatory cells. Again, before vitamin D becomes metabolically active and executes its action, it runs a long journey from absorption (intestine and bile), hydroxylation (liver and kidney) to binding with receptors (receptor polymorphism). In critically ill children the various stages of vitamin $\mathrm{D}$ pathway are compromised and the assessment of the exact defect is not always possible. In the light of above discussion, whether administration of a single dose of vitamin $\mathrm{D}$ in critically ill children during the time of admission would improve their outcome also needs to be considered.

\section{Compliance with Ethical Standards}

Conflict of Interest None.

Source of Funding None.

\section{References}

1. Hewison M. Vitamin D, and the intracrinology of innate immunity. Mol Cell Endocrinol. 2010;321:103-11.

2. Prasad S, Raj D, Warsi S, Chowdhary S. Vitamin D deficiency and critical illness. Indian J Pediatr. 2015;82:991-5.

3. Satheesh P, Verma S, Singhi S, Bansal A. Prevalence of vitamin D deficiency among children with sepsis, its association with sepsis severity and its outcome in a pediatric ICU. Crit Care. 2013;17:35.

4. Nabeta HW, Kasolo J, Kiggundu RK, Kiragga AN, Kiguli S. Serum vitamin $\mathrm{D}$ status in children with protein-energy malnutrition admitted to a national referral hospital in Uganda. BMC Res Notes. $2015 ; 8: 418$. 